\section{Diffracted Light Contrast: Improving the Resolution of a Basic Light Microscope by an Order of Magnitude}

W. Barry Piekos

Yale University, New Haven, CT

barry.piekos@yale.edu

\section{Introduction}

The discovery that the diffracted light from a convex edge can be used to form a very high-quality, shadowcast image on any light microscope has led to a device and method, diffracted-light contrast (DLC), which will allow shadowcast imaging to be routinely performed on student/laboratory microscopes(Piekos, 1999, 2003). The surface lattice of Surirella gema was easily resolved, and micrographs comparing the subcellular details of buccal epithelial cells viewed with DLC vs. Nomarski DIC showed that, on the microscopes used, DLC was superior in both the detail it rendered and depth of field. Although the images presented revealed DLC to be an excellent technique, the full capabilities of the technique were not known at the time.

DLC differs from the centuries-old technique of oblique illumination in that it uses an edge plate, inserted at the level of the field stop, as a source of partially coherent, diffracted light while oblique illumination uses some sort of beam stop to create an off-axis beam of illumination (Wright, 1913; Ott, 1924; Diggins, 1940). The shape of the edge has a great influence on the quality of the DLC image with a convex edge generating the highest contrast, most threedimensional image (Piekos, 1999). This observation indicates that the edge itself is actively affecting the beam, via diffraction, and it is this light which is generating the image.

The use of diffracted light as a source of illumination is the basis of schlieren imaging-a common technique used to image refractive, phase-altering samples and one which has, in the past, been applied to light microscopes (Saylor, 1935; Axelrod, 1980). A schlieren apparatus generally consists of two components: a slit aperture, used to generate the Fraunhofer diffraction pattern, and a second beam stop, or knife, inserted into a conjugate plane and used to eliminate zero-order light, thereby providing a darkened background. DLC differs from this common arrangement in that it uses a single convex edge in order to produce the diffraction pattern and it does not require a second knife. With DLC, the coherent rays produced by the single edge will not interfere with rays from an opposing edge, as is the case when using a slit aperture.

When using a convex edge to generate the diffracted light, each point along the edge serves as a point source of diffracted light. Consequently, the sample is actually being illuminated by an infinite number of separate, partially coherent rays, each approaching the specimen from a slightly different direction and interacting with the sample (phase shifting) in a slightly different way (Piekos, 2003). Interference of these rays only occurs at the image plane and will result in a subtle pattern of interference, which, in turn, gives rise to the delicate, graded pattern of shadowing and a quasi-three dimensional appearance. Consequently, DLC appears to be a form of multiple-beam interference-a technique widely used in interferometry and known to give much more precise interference patterns than is achievable with standard two-beam interference optics.

\section{Materials and Methods}

All images contained in this report were made with a standard Leitz Diaplan microscope (ca. 1985) using either a 40/0.70 or 100/1.32 PL Fluotar lens. The microscope was set to its most basic brightfield configuration, i.e., polarizers, DIC prisms and analyzers were removed and both the field stop and condenser iris diaphragms were left wide open. The microscope was fitted with a small diffracting edge plate, located at the level of the field stop, and this was used as previously described (Piekos, 1999). The edge plate is a circular, opaque plate ( $\sim 40 \mathrm{~mm}$ diameter) that is inserted into the optical path until a convex edge is visible within the field of view. The condenser is then defocused from its usual Koehler position so that a broad band of diffracted light (the defocused edge of the edge plate) is cast onto the specimen plane. Excellent
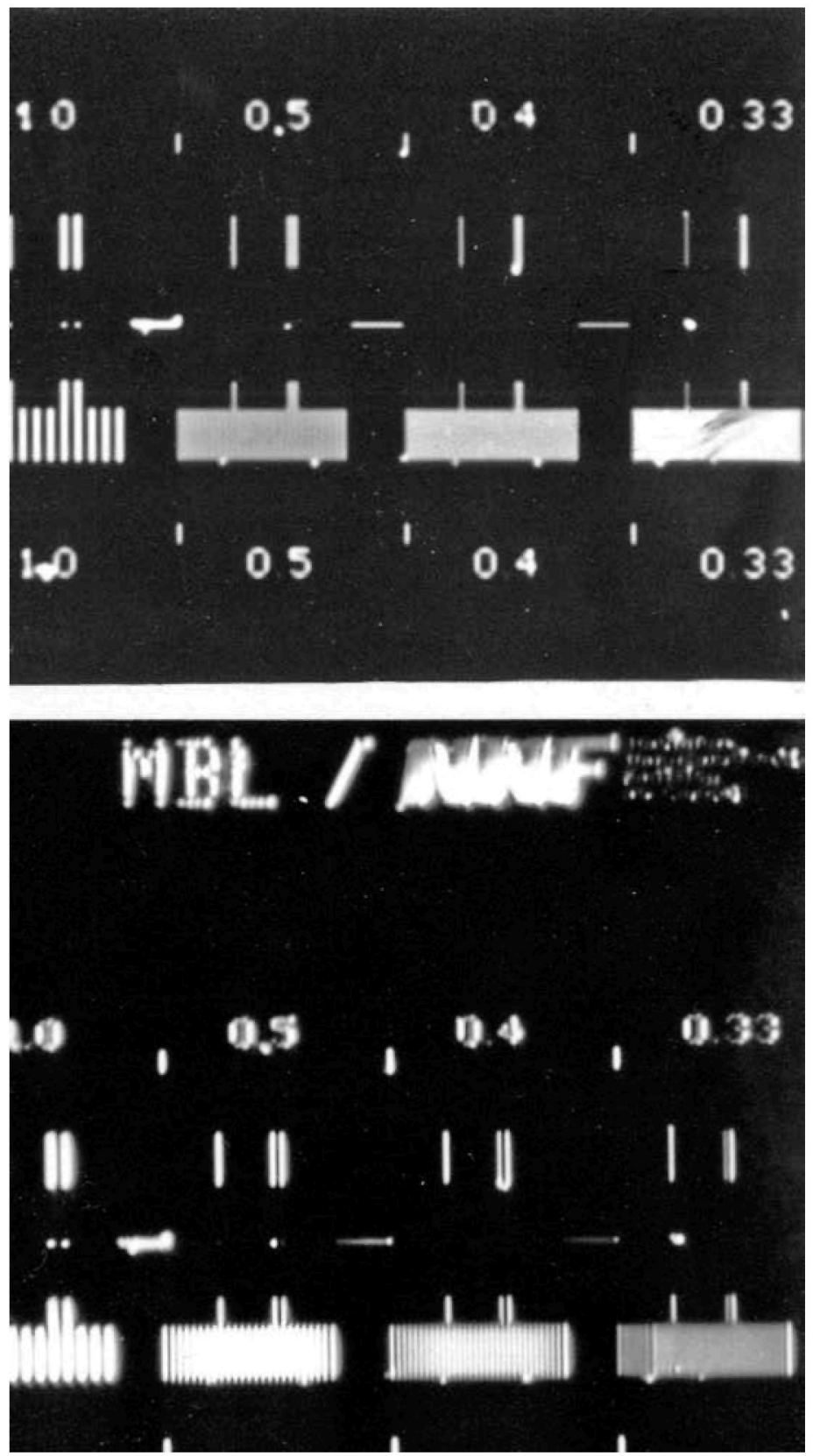

Figure 1. MBL/NNF resolution standard photographed with microscope configured to Koehler illumination (top) and DLC (bottom). Resolution of this purely diffracting sample is clearly better with DLC and exceeds the Abbe limit for the lens used $(1.22 \lambda / 2 \cdot N . A .=480 \mathrm{~nm})$. Leitz 40/0.70 Pl Fluotar lens, 550-nm light. 
Now available with $127 \mathrm{eV}$ of 100,000 counts per second guaranteed

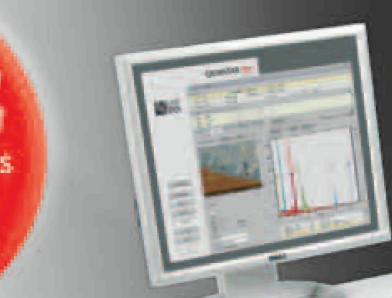

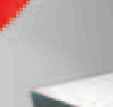
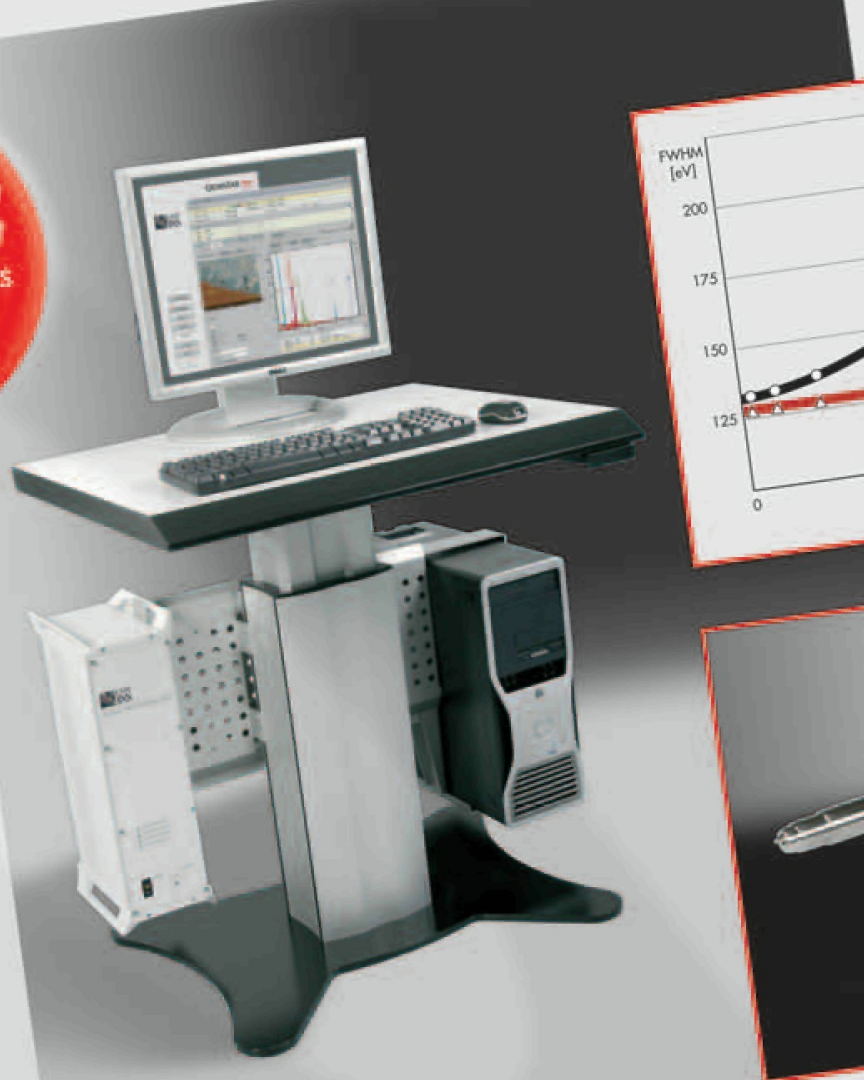

1

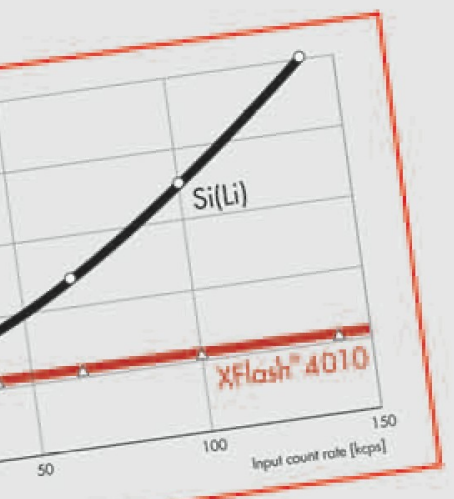

0
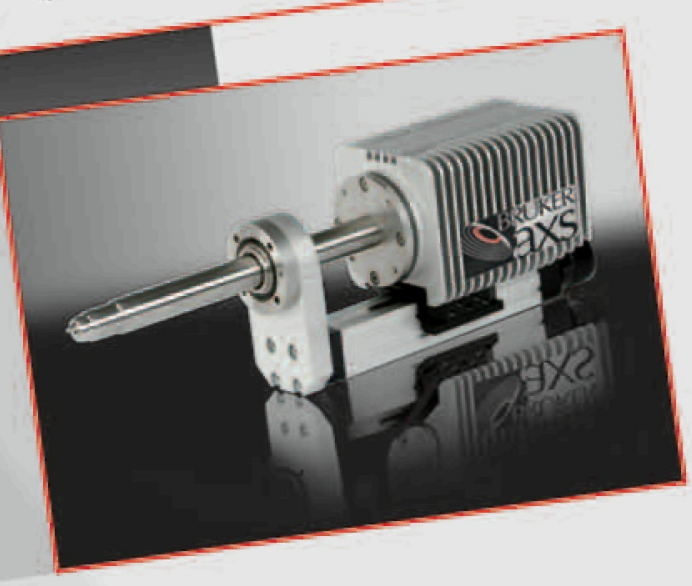

QUANTAX - Taking microanalysis into the fast lane

$\mathrm{NW}_{2}$ free vibration free

See us at the M\&M 2006.

RÖNTEC and PGT

Microanalysis users: Visit the new technologalysis Bruker AXS Microanalysis at booth \# 622 to exp.

4th generation $\mathrm{LN}_{2}$ free XFlash ${ }^{\circledR}$ silicon drift detector

- Unmatched acquisition speed

- Excellent energy resolution at any count rate

- Real time spectroscopy for instar

- Precise light element andysis

- Compatibility with all microscopes software

Modular, customizable ESP

QUANTAX first hand.

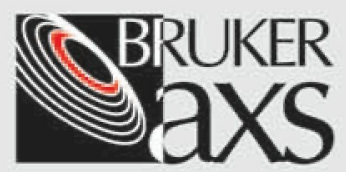


contrast is generated in this region. All micrographs presented in this paper were made with a green filter $(550 \mathrm{~nm})$ and the images were recorded on Kodak Technical Pan Film.

\section{Results and Discussion}

The super-resolving capabilities of DLC are seen in Fig 1. The $\mathrm{MBL} / \mathrm{NNF}$ resolution standard was photographed using the $40 / 0.70$ lens and $550-\mathrm{nm}$ light (theoretical best resolution $=1.22 \lambda / 2 \cdot \mathrm{N}$. A. $=480 \mathrm{~nm}$ ). With Koehler illumination, the $500-\mathrm{nm}$ resolution pattern is faintly visible whereas DLC easily resolves the $500-\mathrm{nm}$ and $400-\mathrm{nm}$ patterns. The elongated $330-\mathrm{nm}$ double-lines are also resolved with DLC. The theoretical resolution of a periodic array of rectangular slits, such as used in the MBL/NNF resolution standard, is $\lambda / 2 \cdot$ N.A. $=390 \mathrm{~nm}$. Consequently, DLC has improved the resolution such that the diffraction-limiting effects of a circular lens used with Koehler illumination $(1.22 \lambda / 2 \cdot N$.A.) has now been superseded by the less-limiting effects of the rectangular sample $(\lambda / 2 \cdot N . A$.$) . The diffraction-limiting effects of the objective lens$ appear less a physical law and more a by-product of Koehler's method of illumination.

Further proof of the resolution-enhancing capabilities of DLC can be seen in Fig. 2. A suspension of 300 $\pm 6-\mathrm{nm}$ and $50 \pm 2-\mathrm{nm}$ Nanospheres (Duke Scientific Corporation) was evenly spread on a microscope slide, allowed to dry, and photographed using the same 40/0.70 lens. Both single 300-nm spheres as well as individual 50$\mathrm{nm}$ spheres are visible. When greatly enlarged (final magnification $\sim 80,000 \mathrm{X}$ ), two closely opposed $50-\mathrm{nm}$ spheres are distinguished as two small circles of equal size. The diffraction-produced images of the 300-nm spheres do appear anisotropic and given the asymmetry of the illuminating beam this would be expected. Such distortions do not appear significant in images generated using the phase-altering properties of many biological samples (see, for example, the images comparing cytoplasmic details viewed under DLC and DIC in Piekos, 1999).

The optical sectioning capabilities of DLC are shown in Fig. 3. These are sections, taken at $\sim 0.5-1 \mu \mathrm{m}$ intervals, through the nucleus of a living buccal epithelium cell. Of particular interest are Figs. 3B and 3E. Fig. 3B shows a section taken just below the top (coverslip side) of the nucleus. At this level, both portions of the

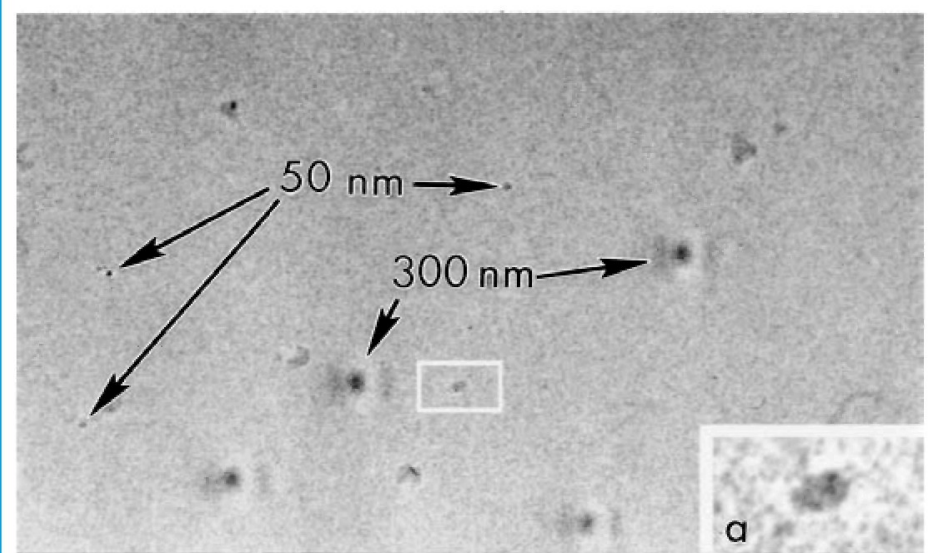

Figure 2. DLC images of 300-nm and 50-nm Nanospheres. When the area defined by the small white rectangle is digitally scanned $(9,400$ dpi) and enlarged to a magnification of $\sim 80,000 x$, the 50-nm spheres are seen as two circles of equal size that are clearly touching (inset a). The resolution of this image is an order of magnitude better than the Abbé limit predicted for the lens used $(480 \mathrm{~nm})$. Leitz 40/0.70 Pl Fluotar lens, 550-nm light. nuclear interior and exterior can be seen and nuclear pores can be detected on the exterior of the nucleus. Fig. $3 \mathrm{E}$ is even more impressive for now the back curvature of the nucleus is in view. A small exterior portion of the nucleus is visible but primarily the inside of the nucleus is visible and nuclear pores are detected from the inside. These optical sections, although very thin, are strikingly shadowed and three-dimensional, an effect best obtained with a convex edge. Enlargement of Fig. 3E to a final magnification of $\sim 70,000 \mathrm{X}$ reveals a macromolecular structure that appears similar to a nuclear pore complex (NPC). A central pore, peripheral proteins, and a portion of the interior nuclear basket are easily visible (Fig. 3F). The presence of this basket suggests the NPC-like structure is being viewed from inside the nucleus.

Proof that this is a nuclear pore complex and not some other, previously undiscovered, ring-like structure associated with the nuclear envelope must await more definitive molecular labeling and/or quantitative studies. The structure does appear to be approximately twice as large as most textbook values given and this fact may argue against it being an NPC. Alternatively, since NPCs have previously only been seen in prepared samples (i.e., fixed, frozen, or mechanically disrupted cells), and never in a living cell, it may be that the previously accepted size of an NPC $(\sim 100 \mathrm{~nm})$ has been artifactually produced and is smaller than the true size. However, for the purposes of the present study, the figure does show that it is possible to image macromolecular assemblies on the order of a nuclear pore complex - structures that previously were thought beyond the capabilities of a basic light microscope.

When a photographic negative is enlarged to an extreme degree (219 times in the case of Fig. 3F), aggregations of silver grains in a film emulsion can sometimes generate patterns that may be falsely interpreted as a real image. Both the negatives and final prints of Fig. 3 were sent to experts at Eastman Kodak Company for analysis. After making their own prints from the Fig. $3 \mathrm{~F}$ negative they conclude that the image shown in Fig. $3 \mathrm{~F}$ is real for two reasons. First, silver grains have never been seen to spontaneously form circles (personal communication with Gary Custozzo, Eastman Kodak Company). Each of the small, ring-like structures, which may be interpreted as peripheral proteins, does not resemble any known pattern of silver complex ever seen in a film emulsion. Second, the fact that a number of these rings, regularly spaced and of the same size, are seen in the same field make it virtually impossible that this image is the result of emulsion artifact. Clearly, this same reasoning can be applied to the circular shapes (50-nm Nanospheres) seen in Fig. 2 a.

The molecular imaging seen in Fig. 3F is likely some form of optical scattering and/or absorption. Scattering of a coherent laser beam can be used to detect 5-nm gold particles but it is not considered to be effective at resolving structures (Cogswell, 1995). However, scattering of partially coherent electrons has always been the basis of the high-resolution images that typify TEM. In Abbé defined microscopy, this high resolution would be attributed to the extremely small wavelength of the electron beam; however, it is clear now that not all forms of optical microscopy necessarily conform to Abbés rules and drawing parallels between TEM and DLC may not be unwarranted. Indeed, it is unlikely that the high-resolution DLC images (Figs. $2 \mathrm{a}$ and $3 \mathrm{~F}$ ) can be easily explained by wave optics and these images may be the first examples of images produced using the quantum properties of light rather than the wave properties. 


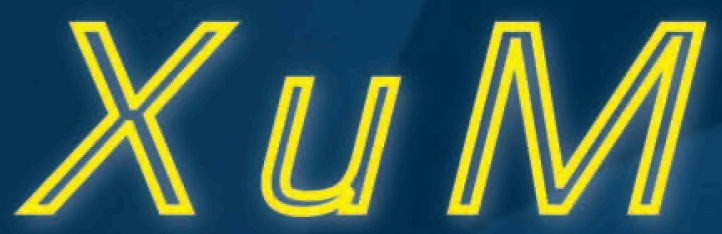

\section{X-ray ultraMicroscopy in the SEM}

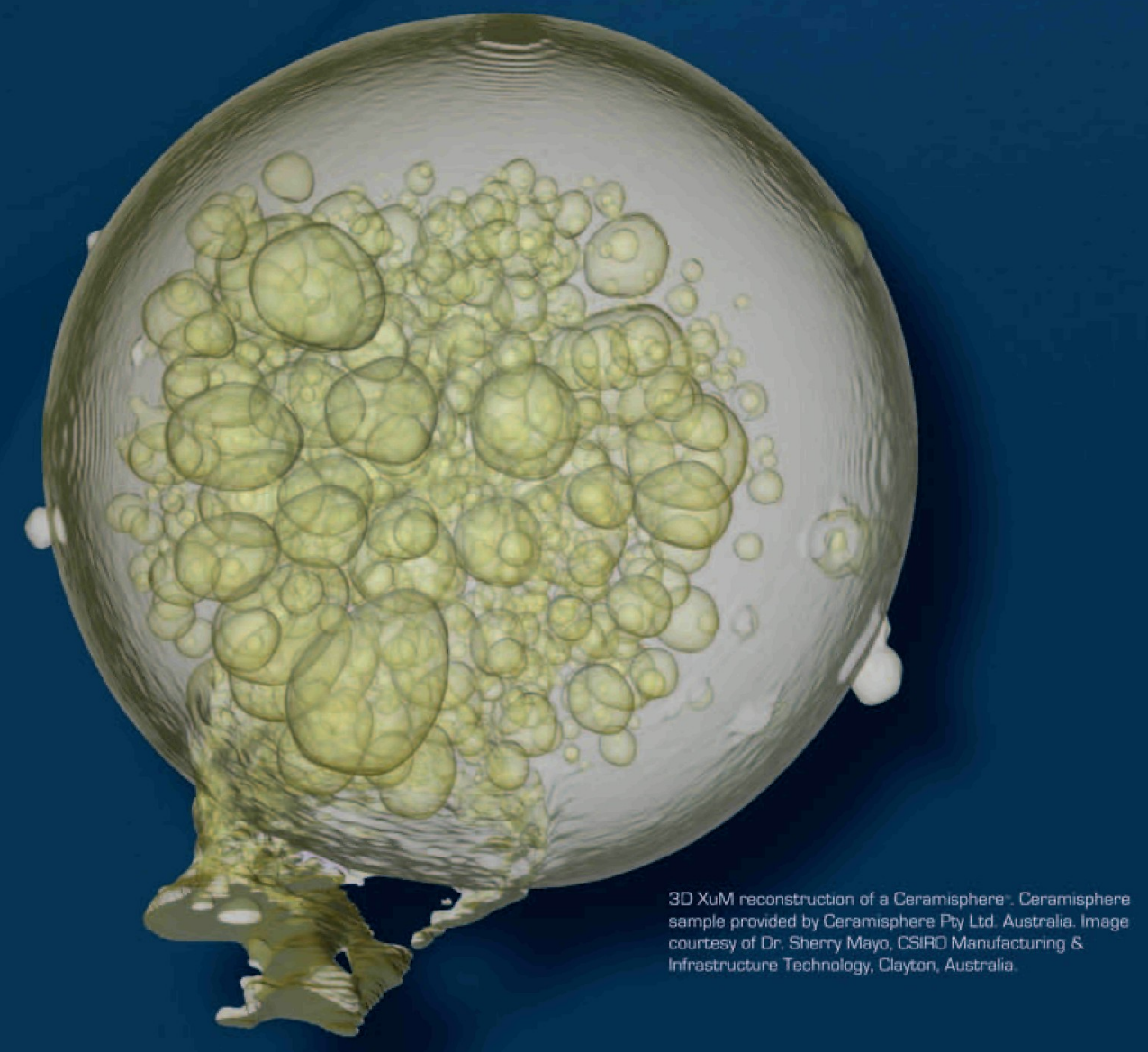

• REVEAL INTERNAL STRUCTURE • HIGH RESOLUTION

• PHASE AND ABSORPTION CONTRAST • 3D MICRO-TOMOGRAPHY
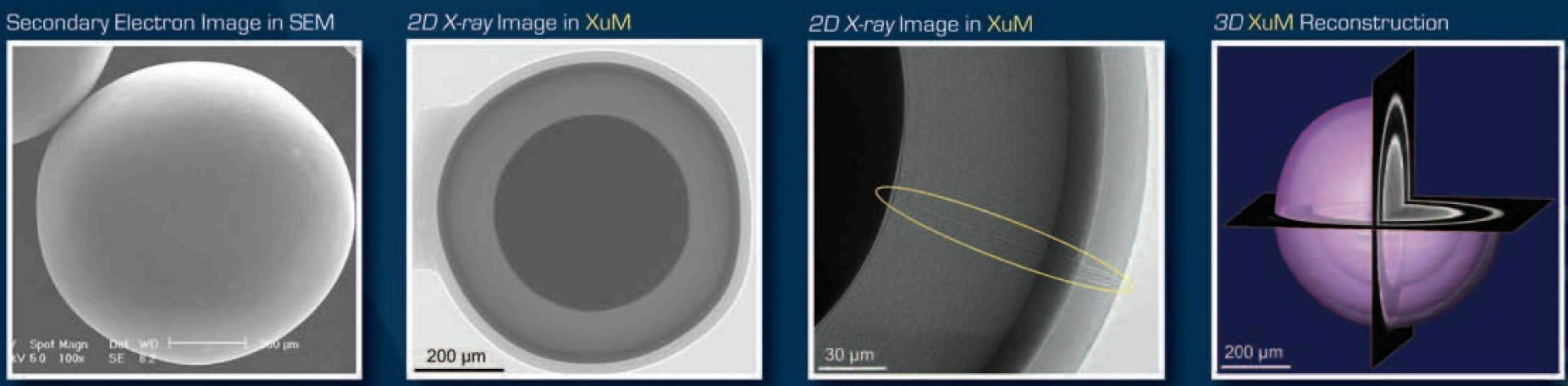

Images: SEM and XuM imaging of an intact multileyered micro sphere. From left to right: Conventional secondary electron image: $2 D \mathrm{XuM}$ image showing composite internal structure; $2 \mathrm{D}$ XuM image magnified highlighting fracture showing the complementary nature of phage and absorption contrast; 3D XuM tomographic recanstruction with cutaway section to expose internal core.

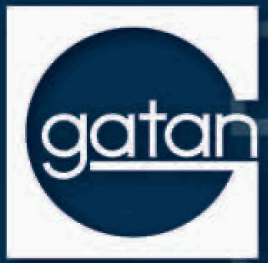


To date, efforts to image the periodicity within individual molecules, e.g., the 67 -nm periodicity of type I collagen, have proven unsuccessful. The difficulty may not be one of resolution because Figs. $2 \mathrm{a}$ and $3 \mathrm{~F}$ would certainly suggest DLC is capable of generating sufficient resolution. Instead, it would seem that contrast may be the limiting factor.

DLC may circumvent the Abbé limit for two reasons. First, because the effective source of illumination (the convex edge) is placed near the level of the field-stop, the illuminating light path is precisely the same as the image-forming light path. This can be proven by the fact that the defocused edge is always visible through the eyepieces. As a result, the sample truly is being illuminated not by a diffuse beam of illumination, as would be the case in Koehler illumination, but by a precisely ordered pattern of coherent light. That pattern can be adjusted by altering the shape and radius of the diffracting edge. It is possible to empirically verify that patterns of partially coherent diffracted light are transmitted through the microscope simply by replacing the single edge of the edge plate by a narrow slit aperture. When such an aperture is inserted at the level of the field stop and the condenser is defocused from its normal Koehler position, interference bands form between the two edges of the slit, indicating the presence of coherent, diffracted light, and these interference bands may be viewed at all levels of the microscope (specimen plane, back aperture of the objective lens, etc.). Of course, the transmission of the single-edge diffracted light and its use in forming the DLC image does not preclude the notion that oblique illumination is also contributing to the image.

Second, when in DLC and because the condenser is defocused from its usual Koehler position, the image of the diffracting edge formed at the back-focal plane of the objective lens is in relatively sharp focus. This is very different from Koehler illumination which gives a diffuse, highly-diffracted representation of the edge at the back-focal plane-the result of diffraction as light from the sample passes through the lens. Consequently, DLC retains the spatial characteristics of the original, diffracted-light illuminating beam, and the information it carries, even though the light and image have passed through the objective lens.

The current interest in super-resolution optical microscopy has led to the development of sophisticated opto-electronic microscopes (e.g., scanning laser confocal and scanning near-field optical microscopes) that can enhance the resolution of a microscope beyond that approximated by Rayleigh's criterion (Schrader et al., 1998; Gustafsson et al., 1999; de Lange et al., 2001; Watanabe et al., 2003). The development of solid immersion, high-numerical aperture lenses are a way of pushing the Abbé limit to its extreme. DLC is a decidedly low-tech way of achieving super-resolution to an extent that has never been seen on any optical microscope. The very existence of the images presented in this report challenges Abbés conclusion that the ultimate resolution of a basic light microscope is, or perhaps ever was, $\sim 200 \mathrm{~nm}$ as dictated by diffraction-limited optics or that it is the objective lens that is primarily responsible for limiting the resolution of a microscope. The method of illumination may be as, or even more, important in determining ultimate resolution and Koehler illumination is not necessarily the best configuration for optimum resolution.

\section{Summary}

DLC is a simple, low-cost method of generating a high-quality, shadowcast image on student/clinical microscopes thereby mak-
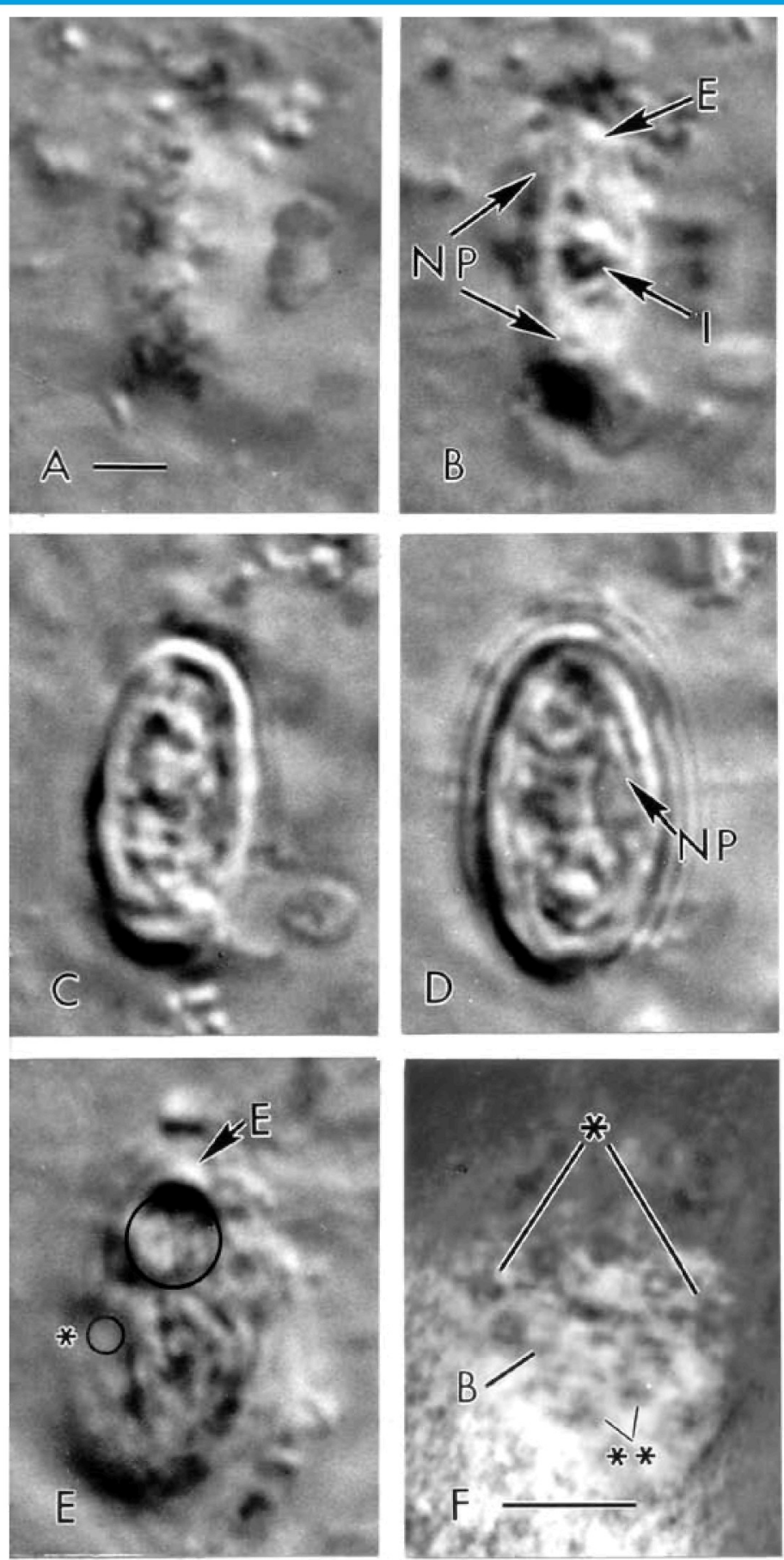

Figure 3. Optical sections through the nucleus of a buccal epithelium cell. Series starts at the top (coverslip side) of the nucleus (A). A slight bulge is seen where the mucleus is protruding into the cytoplasm. $\mathrm{B}$, Section just grazes the surface of the nucleus exposing a small portion of the interior (I). Most of the section reveals nuclear exterior (E), which is covered with nuclear pores (NP). E, Back curvature of the nucleus. A small exterior portion $(E)$ is visible but most of the section reveals internal structure. Nuclear pores are visible on the internal surfaces (within large circle) and these pores appear to be connected by fine lines (elements of nuclear lamina?). All sections clearly show nuclear compartments. Area defined by small circle (asterisk) contains a ring-like structure, which appears very similar to a nuclear pore complex (NPC, F). F, Major molecular components of NPC are clearly visible with eight peripheral proteins circling central pore and a portion of the internal nuclear basket (B). The pore appears slightly elongated along one axis and the peripheral proteins lying along that axis (asterisk) appear further from the center than other proteins. Two of the peripheral proteins appear divided into vaguely $M$-shaped subunits (double asterisk). Leitz 100/1.32 PL Fluotar oil lens; $\mathbf{A}-\mathbf{E}$, scale bar $(\operatorname{shown}$ in $\mathbf{A})=2 \mu \mathrm{m} ; \mathbf{F}$, scale bar $=200 \mathrm{~nm}$. 
ing it possible to routinely introduce this type of imaging into the classroom. However, it is now clear that DLC is easily capable of purely optical resolution at least an order of magnitude better than that produced by diffraction-limited techniques, without the use of lasers, or video or computer enhancement. In addition, it has optical sectioning capabilities that compare favorably to those of laser scanning confocal microscopy. This makes possible the development of much higher magnification basic light microscopes and the direct, in vivo, real-time imaging of exceedingly small structures, such as subnuclear compartments or nuclear pore complexes-structures, which, in the past, have been investigated with fluorescent probes or EM (Gonzalez-Melendi et al., 2000). The fact that these images can be made so simply using a 20 -year-old, off-the-shelf, basic light microscope, equipped with a medium grade set of lenses, challenges Abbés conclusion that the ultimate resolution of a basic light microscope is diffraction-limited to $\sim 200 \mathrm{~nm}$.

\section{References}

Axelrod, D. (1980) Zero-cost modification of bright field microscopes for imaging phase gradient on cells: schlieren optics. Cell Biophys 3, 167-173.

Cogswell, C.J. (1995) In Handbook of Biological Confocal Microscopy, Second Edition (ed. J.B. Pawley). Plenum Press, New York and London, pp. 507-513.

de Lange, F., Cambi, A., Huijbens, R., de Bakker, B., Rensen, W., Garcia-Parajo, M., van Hulst, N. \& Figdor, C.G. (2001) Cell biology beyond the diffraction limit: near-field scanning optical microscopy. I Cell Sci, 114, 4153-4 160.

Diggins, B.A. (1940) Oblique light diaphragm. U.S. Patent 2,195,166.

Gonzalez-Melendi, P., Boudonek, K., Abranches, R., Wells, B., Dolan, L. \& Shaw, P. (2000) The nucleus: a highly organized but dynamic structure. J Microscopy 198(3), 199-207.

Gustafsson, M.G.L., Agard, D.A. \& Sedat, J.W. (1999) I ${ }^{5}$ M: 3D widefield light microscopy with better than $100 \mathrm{~nm}$ axial resolution. J. Microscopy 195(1), 10-16.
Ott, H.N. (1924) Oblique light diaphragm. U.S. Patent 1,503,800.

Piekos, W.B. (1999) Diffracted-light contrast enhancement: a re-examination of oblique illumination. Micros Res Tech 46, 334-337.

Piekos, W.B. (2003) Apparatus and method for producing diffracted light contrast enhancement in microscopes. U.S. Patent 6,600,598.

Saylor, C.P. (1935) Accuracy of microscopical methods for determining refractive index by immersion. J Res Nat Bur Standards 15, 277-294.

Schrader, M., Hell, S.W. \& van der Voort, H.T.M. (1998) Three-dimensional superresolution with a $4 \mathrm{Pi}$-confocal microscope using image restoration. J Applied Physics 84(8), 4033-4042.

Watanabe, T., Iketaki, Y., Omatsu, T., Yamamoto, K., Sakai, M. \& Fujii, M. (2003) Two-point-separation in super-resolution fluorescence microscope based on up-conversion fluorescence depletion technique. Optics Express 11(24), 3271-3276.

Wright, F.E. (1913) Oblique illumination in petrographic microscope work. Am J Sci 205, 63-82.

\section{Acknowledgments}

The loan of the MBL/NNF resolution standard by Dr. Shinya Inoué (Marine Biological Laboratory) is greatly appreciated. Dr. Rudolf Oldenbourg (Marine Biological Laboratory) kindly provided helpful comments concerning the manuscript. I thank Dr. Susan Wente (Vanderbilt University) and Dr. Richard Wozniak (University of Alberta) for their helpful correspondence concerning the interpretation of Fig. 3F. Thanks also to Gary Custozzo and Peter Vimislik (Eastman Kodak Company) for their technical analysis of the Fig. 3F negative and print. Credit, and thanks, must be given to the artisans at Atlantic Film Works (Hamden, CT) for enlarging the Fig. 3F negative.

\section{STOP HASSLING WITH
MULTIPLE SERVICE CONTRACTSI}

START by putting all of our instruments under one service contract with MAS (regardless of make or model). Our expert EM SERVICE GROUP has the knowledge and skills to keep your instrument working at its best.

\section{TEM'S / SEM'S PREP EQUIPMENT SPECIAL SERVICES}

HITACHI TOPCON JOEL ISI AMRAY CAMBRIDGE

\section{VACUUM COATERS PLASMA ASHERS SPUTTER COATERS ION MILLS}

STAGES

BEAM BLANKERS CUSTOM DEVICES

\section{NEW NEW NEW}

\section{Authorized service representatives}

for Gatan preparation equipment

and Topcom TEM'S.
Contracts and On-Demand Emergency Service at Reasonable Rates from Factory Trained Specialist

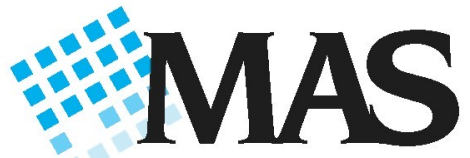

\section{0-421-8451}

3945 Lakefield Court Suwanee, Georgia 30024 770-866-3200 FAX 770-866-3259 616 Hutton Street Suite 101 Raleigh, North Carolina 27606 919-829-7041 FAX 919-829-5518 ADVANGED ANALYTICAL PRODUGTS AND SERVIGES 